\title{
Special Issue on Second International Conference on the Theory and Practice of Natural Computing, TPNC 2013
}

\author{
Adrian-Horia Dediu $^{1}$ - Carlos Martín-Vide ${ }^{1}$
}

Published online: 28 November 2015

(C) Springer-Verlag Berlin Heidelberg 2015

This special issue of the journal 'Soft Computing - A Fusion of Foundations, Methodologies and Applications' offers extended versions of some of the best papers presented at the Second International Conference on the Theory and Practice of Natural Computing, TPNC 2013, held in Cáceres, Spain, on December 3-5, 2013, under the organisation of the Research Group on Mathematical Linguistics (GRLMC) from Rovira i Virgili University in Tarragona, Spain and the Computer Architecture and Logic Design Group (ARCO) from University of Extremadura, Spain.

TPNC 2013 was the second event in a series dedicated to host and promote research in a wide spectrum of computational models, methods and techniques inspired by information processing in nature. We encouraged both theoretical contributions in soft computing, computing architectures, and formal models as well as solutions for practical problems based on natural computing methods.

Out of 47 submissions to the conference, 18 papers were accepted (which represents a competitive acceptance rate of about $38 \%$ ). Among them, the authors of 13 papers were invited to submit to this special issue. Each submission was reviewed by at least two experts and, on the basis of their comments, the guest editors decided to accept six papers for this special issue (which represents an acceptance rate of about $13 \%$ out of the submissions to the conference).

Adrian-Horia Dediu

adrianhoriadediu@yahoo.com

1 Research Group on Mathematical Linguistics (GRLMC), Rovira i Virgili University, Av. Catalunya, 35, 43002

Tarragona, Spain
The selected papers cover a broad spectrum of topics, whose main achievements are summarized now.

In the paper Possibilistic AIRS Induction from Uncertain Data, Rim Hentech, Ilyes Jenhani, and Zied Elouedi propose a new classification approach combining AIRS and the possibility theory. In the new approach, the uncertainty is expressed via possibility distributions. The experiments presented show the improvements of the new method.

Lingling Jin and Ian McQuillan propose three theoretical models based on strings, matrices, and context-free grammars for DNA sequences that can either move or copy themselves to new positions within a genome. In their paper Computational Modelling of Interruptional Activities between Transposable Elements using Grammars and the Linear Ordering Problem, the authors discuss the advantages and limitations of each model.

The paper Analysis of Long-term Swarm Performance based on Short-term Experiments, by Yara Khaluf, Mauro Birattari, and Franz Rammig, proposes an application of the Central Limit Theorem. Based on a well-known established theory, the authors design several experiments, showing that the results are concordant with the ones predicted by the theory.

Mirosław Kordos and Andrzej Rusiecki, in Reducing Noise Impact on MLP Training-Techniques and Algorithms to Provide Noise-Robustness in MLP Network Training, describe and implement several state-of-the-art methods and propose various new ones. The general conclusion from the work is that network training methods should be adjusted to the amount of noise in the data.

In the paper Studying the Multiobjective Variable Neighbourhood Search Algorithm when Solving the Relay Node Placement Problem in Wireless Sensor Networks, the authors Jose M. Lanza-Gutierrez, and Juan A. Gomez-Pulido study how to deploy relay nodes into previously established static 
traditional Wireless Sensor Networks, with the purpose of optimising two important features: the average energy consumption of sensors and the average sensitivity area. The results show that the Multiobjective Variable Neighbourhood Search Algorithm (MO-VNS) performs better than the standard evolutionary algorithms.

In their paper Quantum-based Secure Communications with no prior Key Distribution, Marius Nagy and Naya Nagy present a series of protocols that are entanglement free and use only unary quantum transformations. The computational power assumed is less than that of a universal quantum computer; therefore, using the quantum mechanics to directly encrypt the transmitted message into a sequence of qubits leads to a simple, fast and more efficient method of communication.

We thank all reviewers for their valuable work. We also thank all the authors for their contributions as well as the editorial team of the journal for their hard work.

\section{External reviewers}

Nizar Bouguila

Rui Camacho

Shyamala Doraisamy

Moumen T. El-Melegy

Simon J. Gay
Laszlo Gyongyosi

Vladik Kreinovich

Lei M. Li

Yun Liang

Mark W. Maimone

Peter Marwedel

Gilles Mauris

Catherine Meadows

Patricia Melin

Risto Miikkulainen

Sang-Hoon Oh

Yew-Soon Ong

Gary B. Parker

José Miguel Puerta

Dylan A. Shell

Kasper Støy

Thomas Stuetzle

Javid Taheri

Himanshu Tyagi

Andrew Vardy

Fatos Xhafa

Wenfu Xu

Toshiyuki Yasuda

Mark Yim

Kazuya Yoshida

Yi Zhang 